\title{
Isolation, identification, culture and production of adenosine and cordycepin from cicada larva infected with entomopathogenic fungi in Thailand
}

\author{
Aphidech Sangdee $^{1 \star}$ and Kusavadee Sangdee ${ }^{2}$ \\ ${ }^{1}$ Department of Biology, Faculty of Science, Mahasarakham University, 44150 Thailand. \\ ${ }^{2}$ Preclinical Division, Faculty of Medicine, Mahasarakham University, 44150 Thailand.
}

Accepted 19 December, 2012

\begin{abstract}
In this study, cicada larvae infected with entomopathogenic fungi were collected from Maha Sarakham Province in northeast Thailand. One strain of entomopathogenic fungi with cottony white cream colonies was isolated. Small subunit (SSU) rDNA, large subunit (LSU) rDNA, the elongation factor 1a (EF-1 $\alpha$ ) and the largest subunit of RNA polymerase II (rpb1) sequence analyses were used for identification of the isolate. A BLAST search in NCBI showed the sequence to be most similar to Cordyceps sp., Hirsutella sp., Ophiocordyceps longissima and O. Iongissima for SSU rDNA, LSU rDNA, EF-1 $\alpha$ and rpb1, respectively. This correlated well with evidence from neighbor-joining trees, based on SSU rDNA, LSU rDNA, EF-1 $\alpha$ and rpb1. Therefore, the isolate was classified as an anamorph strain of Ophicordyceps and assigned as Ophiocordyceps Iongissima isolate Cod-MK1. The O. longissima isolate Cod-MK1 was found to grow best in HCGA medium, compared with six other synthetic media. Moreover, the isolate was shown to develop numerous synnemata (stroma-like stalks) and conidia, when cultured in the applied HCGA medium at 25 to $28^{\circ} \mathrm{C}$ for 60 to 90 days. The content of adenosine was observed only in the extract from dried mycelia at $31.68 \mu \mathrm{g} / \mathrm{g}$. The content of cordycepin from dried mycelia was $335.65 \mu \mathrm{g} / \mathrm{g}$; lower than those of the extract from dried stroma-like stalks $(366.14 \mu \mathrm{g} / \mathrm{g})$. Therefore, the induced culture from this study could be used for the production of adenosine and cordycepin in 0 . longissima isolate Cod-MK1.
\end{abstract}

Key words: Ophiocordyceps sp., identification, culture medium, phylogenetic tree, adenosine, cordycepin.

\section{INTRODUCTION}

The genus Cordyceps is a group of entomopathogenic fungi that form fruiting bodies and live mainly on insects and other arthropods (Wu et al., 2011; Zhu et al., 2011). Many Cordyceps species are parasites of the cicada larvae (Wang et al., 2012). The infection process begins when spores of the fungus geminate and invade the body of the cicada larvae. Consumption of nutrients by the fungal hyphae eventually results in the death of the host. The internal tissue of the cicada larvae becomes

*Corresponding author. E-mail: aphidech_sangdee@yahoo.com. replaced by a mass of mycelium and is transformed into a sclerotium. After that, the fruiting body was developed on the surface of the host (Webster, 1980).

Many fungi belonging to Cordyceps have been used as food and herbal medicines in Asia (Kuo et al., 2005). In China, some Cordyceps spp. have successfully been used in immunity modulation, fatigue resistance, longevity elongation, and other functions ( $\mathrm{Nam}$ et al., 2006). C. sinensis has been widely used as a general tonic for protecting and improving lung and kidney functions (Leung et al., 2009), as a roborant, a sedatives, and a supplementary therapy for jaundice, opiumism, tuberculosis and cancer (Nam et al., 2006). Also, C. 
militaris and C. ohioglossoides have been used in Japan for their anticarcinogenic and immunomodulatory activities (Ohmori et al., 1986; Kiho et al., 1996).

There are reported to be over 300 species of Cordyceps worldwide, more than 80 of which have been identified in Korea (Nam et al., 2006). In the last few years, PCR has successfully been used to systematically investigate Cordyceps species. PCR techniques based on particular regions of the fungal genome have been used for fungal taxonomic grouping, establishing evolutionary relationships, and functional properties (Kuo et al., 2005). Moreover, these techniques have been particularly useful in discriminating fungi at interspecific and intraspecific levels (Matsuda and Takamatsu, 2003; Skouboe et al., 1999).

Biochemical studies of Cordyceps spp. have shown they contain many bioactive compounds with different effects on the human body (Zhu et al., 1998; Weng et al., 2002; Holliday and Cleaver, 2008; Paterson, 2008; Das et al., 2010). Two common difficulties encountered in such studies are the slow growth rate and different morphological characteristics of Cordyceps spp. (Nam et al., 2006). Moreover, the quantity of bioactive compounds and growth rate can vary depending on artificial conditions and Cordyceps species (Liu et al., 2001; Dong and Yao, 2005; Masuda et al., 2006; Hung et al., 2009). Therefore, the present study sought to identify entomopathogenic fungi from cicada larvae in Thailand using the PCR technique with a particular genome, to understand its culture for optimum mycelial growth and conidia production, and to determine adenosine and cordycepin production from this fungus.

\section{MATERIALS AND METHODS}

\section{Isolation of entomopathogenic fungi}

Ten cicada larvae infected with entomopathogenic fungi were collected from mixed deciduous forest, Maha Sarakham province in northeast Thailand. Isolation was carried out using the tissue transplanting technique. The cicada samples were washed with sterile distilled water and sectioned into two pieces. The inner tissue of cicada larvae were cut $\left(5 \times 5 \mathrm{~mm}^{2}\right)$, surface sterilized by dipping in $1 \%$ sodium hypochloride for $2 \mathrm{~min}$, and rinsed several times with sterile distilled water before being transferred onto the surface of potato dextrose agar (PDA). The mycelium growing out of the cicada larvae tissue was sub-cultured on potato dextrose agar (PDA) and incubated at $25-28^{\circ} \mathrm{C}$ for further study.

\section{Identification of entomopathogenic fungi}

The isolate was cultured in PDA at 25 to $28^{\circ} \mathrm{C}$ for 20 days. Mycelia harvested from the PDA were homogenized with liquid nitrogen in a mortar and pestle. The mycelium powder was transferred to a microcentrifuge tube, and genomic DNA was extracted using the plant DNA extraction kit handbook (Vivantis, Malaysia). DNA samples were checked on 1\% agarose gel electrophoresis and stored at $-20^{\circ} \mathrm{C}$.

The partial small subunit of rDNA was amplified using primers NS1 (5'-GTAGTCATATGCTTGTCTC-3') and NS2 (5'GGCTGCTGGCACCAGACTTGC-3') (White et al., 1990). The temperature profiles for the PCR cycles were as follows: initial denaturation at $94^{\circ} \mathrm{C}$ for $4 \mathrm{~min}$, followed by 35 cycles at $94^{\circ} \mathrm{C}$ for 1 $\min , 55^{\circ} \mathrm{C}$ for $1 \mathrm{~min}, 72^{\circ} \mathrm{C}$ for $1.5 \mathrm{~min}$, and a final extension reaction at $72^{\circ} \mathrm{C}$ for $10 \mathrm{~min}$. The partial large subunit of rDNA was amplified by PCR using primers LROR (5'-ACCCGCTGAACTTAAGC-3') and LR7 (5'-TACTACCACCAAGATCT-3') (Vilgalys and Hester, 1990). PCR was performed under the temperature profiles of $94^{\circ} \mathrm{C}$ for 4 min, followed by 38 cycles at $94^{\circ} \mathrm{C}$ for $1 \mathrm{~min}, 47^{\circ} \mathrm{C}$ for $45 \mathrm{~s}, 72^{\circ} \mathrm{C}$ for $2 \mathrm{~min}$, and a final extension at $72^{\circ} \mathrm{C}$ for $10 \mathrm{~min}$. The elongation factor $1 \alpha(E F-1 \alpha)$ was amplified by PCR using primers EF-983F (5'GCYCCYGGHCAYGGTGAYTTYAT-3') and EF-2218R (5'GACTTGACTTCRGTVGTGAC-3') (Currie et al., 2003), and the largest subunit of RNA polymerase II (rpb1) was amplified using primer CRPB1 (5'-CCWGGYTTYATCAAGAARGT-3') and RPB1Cr (5'-CCNGCDATNTCRTTRTCCATRTA-3') (Castlebury et al., 2004). PCR of EF-1 $\alpha$ and rpb1 gene were performed under the same temperature profiles as follows: $95^{\circ} \mathrm{C}$ for $5 \mathrm{~min}$, followed by 35 cycles at $95^{\circ} \mathrm{C}$ for $1 \mathrm{~min}, 55^{\circ} \mathrm{C}$ for $1 \mathrm{~min}$, and $72^{\circ} \mathrm{C}$ for $1 \mathrm{~min}$, and a final extension at $72^{\circ} \mathrm{C}$ for $10 \mathrm{~min}$. The amplification was carried out in a Thermal cycler (Veriti 96-Well Thermal Cycler, Applied Biosystems, USA) in $50 \mu \mathrm{l}$ of polymerase chain reaction (PCR) mixture containing $100 \mathrm{ng}$ of total DNA, $0.2 \mathrm{mM}$ of each dNTP, 5 unit of Tag DNA polymerase (Invitrogen, USA) in $5 \mu$ l of 10x Tag buffer, $1 \mathrm{mM} \mathrm{MgCl}, 0.2 \mathrm{mM}$ of each primer, and $35 \mu$ sterile deionized water. The PCR products obtained were purified with a Gel/PCR DNA fragments extraction kit (Geneaid, USA). Sequencing was performed by using Macrogen Advancing through Genomics (Macrogen Inc., Korea). Sequence data of partial small and large subunit rDNA, EF-1 $\alpha$ and rpb1 gene were compared with sequences in the National Center for Biotechnology Information data bank using the BLAST program (www.ncbi.nih.gov/blast). The novel sequence (JQ922262, JQ922263, JX896449 and JX896450) and reference sequences obtained from GenBank (Table 1) were aligned using ClustalW (www.genome.jp/tools/clustalw/). Phylogenetic analyses were performed using Mega 4 (Tamura et al., 2007) and a Neighbor Joining tree (NJ tree) was constructed (bootstrap replicates $=1000$; seed $=64,238$ ) using the Kimura 2 parameter method for pairwise deletion at uniform rates.

\section{Medium screening for fungal growth}

The synthetic media potato dextrose agar (PDA), Sabouraud dextrose agar (SDA), malt extract agar (MEA), yeast malt agar (YMA), Czapek Dox agar (CDA) and oat meal agar (OMA), and composed medium, homogenized died cricket glucose agar (HCGA), were prepared for culturing the fungal isolate Cod-MK1. The HCGA medium was prepared by dissolving $20 \mathrm{~g}$ homogenized died cricket $1: 2(\mathrm{w} / \mathrm{v})$ in distilled water, adding $3 \mathrm{~g}$ glucose and $1.5 \mathrm{~g}$ agar, then adjusting the final volume to $100 \mathrm{~mL}$. Mycelial disks of the isolate ( $7 \mathrm{~mm}$ in diameter) were cut from colony margins and transferred to the center of each of the seven media. All the inoculated media were incubated at 25 to $28^{\circ} \mathrm{C}$. After 20 days, the colony diameter was measured for mycelial growth. The experiment was performed in triplication to confirm reproducibility of results. Duncan's multiple range test was performed to check the growth differences. Data were assessed using the SPSS software version 14.0.

\section{Induction of synnemata and conidia on applied HCGA medium}

The fungal isolate Cod-MK1 was cultured on HCGA medium at 25 to $28^{\circ} \mathrm{C}$ for 20 days, after which mycelial disks were cut from colony margins and transferred to sterilized applied HCGA medium (containing $20 \mathrm{~g}$ Thai Jasmine rice and $25 \mathrm{~mL}$ homogenized died cricket $1: 2(\mathrm{w} / \mathrm{v})$ in distilled water) in an $8 \mathrm{oz}$ cylindrical bottle. All of the inoculated media were incubated at 25 to $28^{\circ} \mathrm{C}$ and observed daily. 
Table 1. Cordyceps and related species and their NCBI accession numbers used in this study.

\begin{tabular}{|c|c|c|c|c|}
\hline \multirow[t]{2}{*}{ Species } & \multicolumn{4}{|c|}{ NCBI accession number } \\
\hline & SSU & LSU & EF-1 $\alpha$ & Rpb1 \\
\hline Akanthomyces cinereus & - & GQ249971 & - & - \\
\hline Beauveria bassiana & - & EU334677 & - & - \\
\hline Clavicipitaceae sp. LM463 & EF060765 & - & - & - \\
\hline Cordyceps sp. BCC28609 & - & GQ249990 & - & - \\
\hline Cordyceps sp. 97003 & AB027329 & - & - & - \\
\hline Cordyceps cf. acicularis & - & - & - & DQ522371 \\
\hline Cordyceps aphodii & - & - & DQ522323 & - \\
\hline Cordyceps agriota & - & - & - & DQ522368 \\
\hline Cordyceps cylindrica & AF327400 & - & GU979996 & - \\
\hline Cordyceps brunneipunctata & - & - & - & DQ522369 \\
\hline Cordyceps fratricida & - & - & JQ257028 & - \\
\hline Cordyceps gunnii & DQ838789 & - & HM140636 & - \\
\hline Cordyceps heteropoda & - & - & - & AY489651 \\
\hline Cordyceps inegoensis & - & - & DQ118752 & - \\
\hline Cordyceps militaris & AY245671 & - & - & - \\
\hline Cordyceps melolonthae & & - & DQ522331 & - \\
\hline Cordyceps sinensis & AB067701 & $\begin{array}{c}\text { AB067710, AB067711, } \\
\text { AB067737 }\end{array}$ & - & - \\
\hline Cordyceps sobolifera & DQ838796 & - & - & - \\
\hline Cordyceps variabilis & - & - & - & DQ522386 \\
\hline Hirsutella sp. & - & - & GU787114 & - \\
\hline Hirsutella rhossiliensis & - & EF546655 & & - \\
\hline Hirsutella stilbelliformis var. myrmicarum & - & GQ866967 & EU797599 & - \\
\hline Hymenostilbe sp. & - & - & GU797124 & - \\
\hline Hypocrella discoidea & - & DQ384944 & - & - \\
\hline Hypomyces lactifluorum & - & EU710768 & - & - \\
\hline Isaria takamizusanensis & - & - & GU979994 & - \\
\hline Metacordyceps yakusimensis & AB044632 & - & - & - \\
\hline Ophiocordyceps sp. & - & - & GU797121 & - \\
\hline Ophiocordyceps aphodii & DQ522541 & - & - & - \\
\hline Ophiocordyceps gracilis & - & - & EF468750 & - \\
\hline Ophiocordyceps heteropoda & AB084157 & - & EF468752 & - \\
\hline Ophiocordyceps longissima & - & - & EF468757 & EF468865 \\
\hline Ophiocordyceps pulvinata & - & - & - & GU904210 \\
\hline Ophiocordyceps rhizoidea & - & - & EF468765 & $\begin{array}{l}\text { EF468872, } \\
\text { EF468873 }\end{array}$ \\
\hline Ophiocordyceps rubiginosiperitheciata & JN941705 & - & - & - \\
\hline Ophiocordyceps sinensis & - & FJ461354, HM135168 & - & - \\
\hline Ophiocordyceps sobolifera & AB027328, EF468972 & - & - & EF468875 \\
\hline Ophiocordyceps sphecocephala & - & - & - & $\begin{array}{l}\text { JN992431, } \\
\text { JN992432 }\end{array}$ \\
\hline Ophiocordyceps variabilis & - & - & - & EF468885 \\
\hline Paecilomyces sp. SJL0906 & - & HM135165 & - & - \\
\hline Polycephalomyces lilacinus & - & - & GU979988 & - \\
\hline Polycephalomyces formosus & - & AY259544 & - & - \\
\hline
\end{tabular}

\section{Preparation of the entomopathogenic fungi mycelia}

The seed culture of fungal isolate Cod-MK1 was cultured in $50 \mathrm{~mL}$
PDB medium on a rotary shaker at $28^{\circ} \mathrm{C}$ and $150 \mathrm{rpm}$ for 7 days, after which $1 \mathrm{~mL}$ of seed culture was transferred to $25 \mathrm{~mL}$ of the induce culture. The induce culture was prepared by dissolving $0.5 \mathrm{~g}$ 


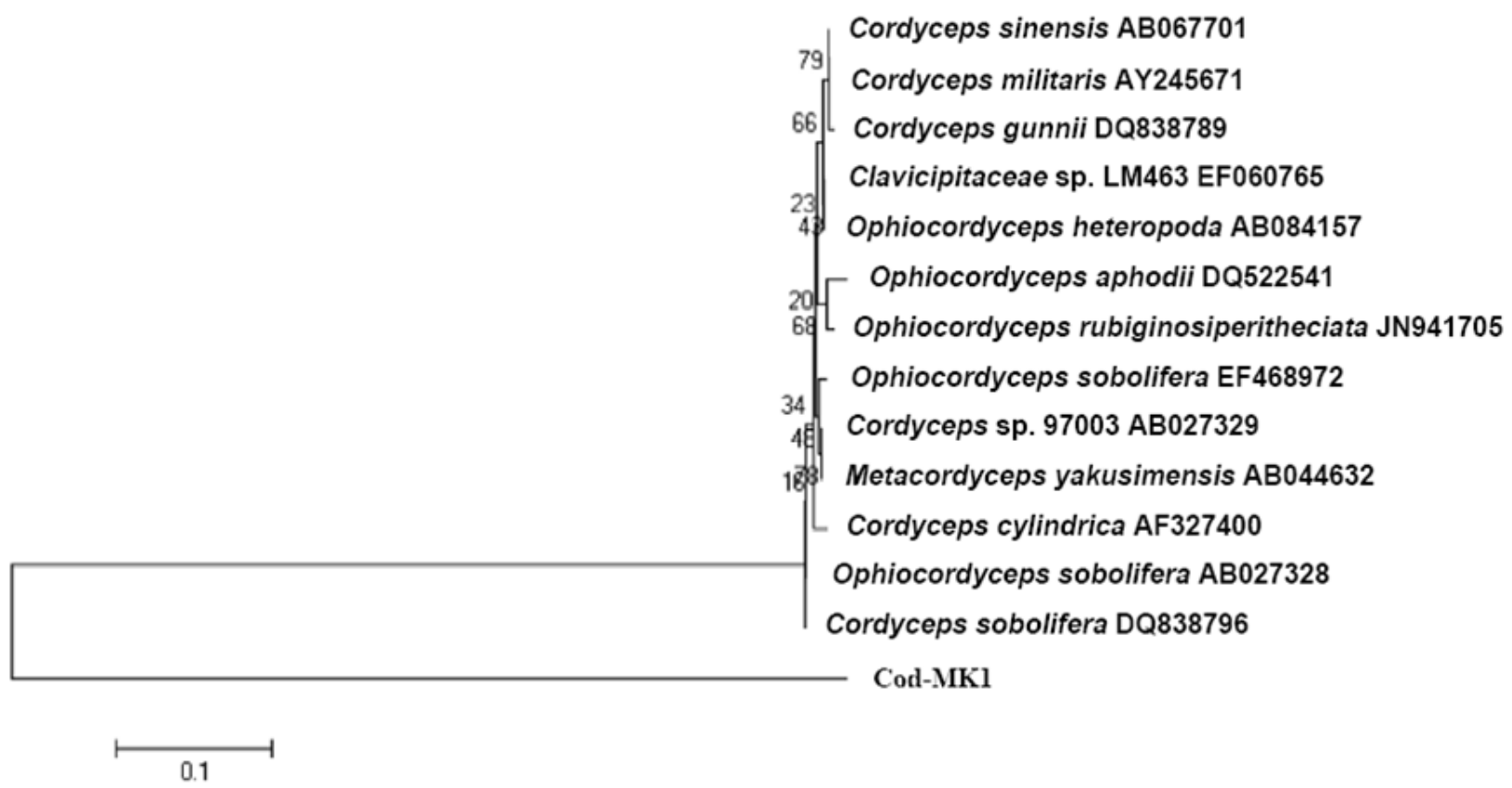

Figure 1. Phylogenetic relationships of the fungal isolate Cod-MK1 with Cordyceps and related species based on partial SSU rDNA sequences. A Neighbor Joining tree ( $\mathrm{NJ}$ tree) was constructed using Mega 4. The percentages expressed above the branches are frequencies with which a given branch appeared in 1000 bootstrap replications.

homogenized died cricket $1: 2(\mathrm{w} / \mathrm{v})$ in distilled water, adding $2 \%$ glucose, $0.9 \%$ yeast extract, $1.5 \%$ peptone, $0.3 \% \mathrm{~K}_{2} \mathrm{HPO}_{4}$, and $0.4 \% \mathrm{CaCl}_{2}$, and then adjusting the final volume to $25 \mathrm{~mL}$. The induce culture was incubated at 25 to $28^{\circ} \mathrm{C}$ without shaking for 14 days. The mycelium on the surface of the induce culture was collected and dried at $50^{\circ} \mathrm{C}$ overnight.

\section{Extraction and determination of adenosine and cordycepin}

The stroma-like stalks and mycelia of the fungal isolate Cod-MK1 were ground into powder. Then, $0.5 \mathrm{~g}$ of fungal isolate MK-1 powder was added into $5 \mathrm{~mL}$ methanol-water (50/50, V/V) in $15 \mathrm{~mL}$ centrifuge tube, followed by sonication with a High Intensity Ultrasonic Processor (Model VCX 750, USA). This was performed on ice for a total of $5 \mathrm{~min}$ in $15 \mathrm{~s}$ bursts with $5 \mathrm{~s}$ gaps for cooling. The sonicated solutions were centrifuged (Tomy MX-301, Japan) at $9100 \mathrm{~g}$ for $5 \mathrm{~min}$ and filtered through a $0.2 \mu \mathrm{m}$ filter prior to HPLC analysis.

Quantities of adenosine and cordycepin were determined by HPLC (Agilent Technologies, Hewlett-Packard-Strasse 8, Waldbronn, Germany) using a Eclipse XDB-C8 column with $150 \times$ $4.6 \mathrm{~mm}$, 5-Micro, diode array detectors (Agilent Technologies, Waldbronn, Geramany). Methanol: Milli-Q water (6:94, V/V) was used as the mobile phase at a flow rate of $1.0 \mathrm{~mL} \mathrm{~min}{ }^{-1}$. The column temperature was kept constant at $30^{\circ} \mathrm{C}$.

\section{RESULTS}

\section{Isolation of entomopathogenic fungi}

The colony on PDA that developed from the inner tissue of cicada larvae grew to $20 \mathrm{~mm}$ in diameter under 25 to $28^{\circ} \mathrm{C}$ within 30 days and assigned as the Cod-MK1 isolate. This isolate produced cottony colonies on PDA with white cream color on the ventral surface and the reverse of the colonies. Mycelium on the medium was tightly, sterile, and 3.5 to $4 \mu \mathrm{m}$ in diameter. Moreover, it produced white cream synnemata or cotton-like hyphae on the cicada larvae tissue.

\section{Identification of the fungal isolate Cod-MK1}

DNA fragments of approximately 600 to $700 \mathrm{bp}$ were amplified by PCR using primers NS1 and NS2. The fragment contained the 5 ' end of small subunit rDNA (SSU rDNA). A fragment of SSU rDNA consisting of 661 nucleotides was submitted to GenBank (accession number JQ922263). A BLAST search in NCBI (www.ncbi.nih.gov/blast) showed this sequence to be most similar to Cordyceps sp. 97003 (AB027329, 95\%), Metacordyceps yakusimensis (AB044632, 95\%), Ophiocordyceps sobolifera (EF468972, 94\%), C. sobolifera (DQ838796, 94\%) and O. sobolifera (AB027328, 94\%). A phylogenetic tree was generated from 14 aligned sequences with an equal character. This tree showed that the fungal isolate did not locate in the same clade with other reference fungal sequences (Figure 1). However, the fungal isolate sequence was closely related with $C$. sobolifera and $O$. sobolifera.

DNA fragments of approximately $1400 \mathrm{bp}$ were amplified by PCR using primers LROR and LR7. The fragment contained the $5^{\prime}$ end of large subunit rDNA 


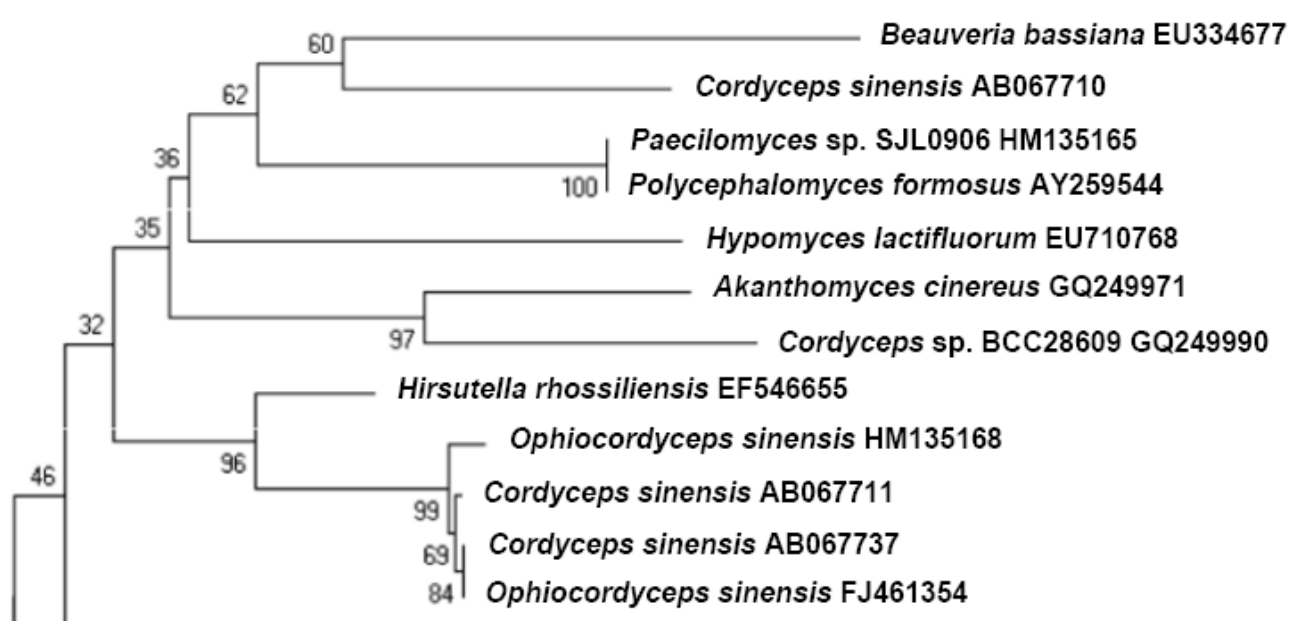

Hypocrella discoidea DQ384944

Hirsutella stilbelliformis var. myrmicarum GQ866967 Cod-MKl

0.01

Figure 2. Phylogenetic relationships of the fungal isolate Cod-MK1 with Cordyceps and related species based on partial LSU rDNA sequences. A Neighbor Joining tree (NJ tree) was constructed using Mega 4. The percentages expressed above the branches are frequencies with which a given branch appeared in 1000 bootstrap replications.

(LSU rDNA). A fragment of LSU rDNA sequence consisting of 1381 nucleotides was submitted to GenBank (accession number JQ922262). The BLAST search in NCBI indicated that the fungal isolate had high sequence similarity with Hirsutella rhossiliensis (EF546655, 93\%), C. sinensis (AB067711, 93\%), O. sinensis (HM135168, 93\%), H. stilbelliformis var. myrmicarum (GQ866967, 93\%), and C. sinensis (AB067704, 93\%). A phylogenetic tree was generated from 15 aligned sequences with an equal character. This tree indicated that the fungal isolate was located in the same clade with $H$. stilbelliformis var. myrmicarum anamorph strain (Figure 2).

DNA fragments of approximately $600 \mathrm{bp}$ were amplified by PCR using primers EF-983F and EF-2218R. A fragment of EF-1a sequence consisting of 517 nucleotides was submitted to GenBank (accession number JX896449). The BLAST search in NCBI indicated the fungal isolate had high sequence similarity with $O$. longissima (EF468757, 99\%), C. aphodii (DQ522323, 93\%), O. rhizoidea (EF468765, 93\%), C. cylindrica (EF468786, 93\%) and C. fratricida (JQ257028, 93\%). A phylogenetic tree was generated from 17 aligned sequences with an equal character. This tree indicated the fungal isolate was located in the same clade as $O$. longissima (Figure 3).

DNA fragments of approximately $750 \mathrm{bp}$ were amplified by PCR using primers CRPB1 and RPB1Cr. A fragment of rpb1 sequence consisting of 654 nucleotides was submitted to GenBank (accession number JX896450). The BLAST search in NCBI indicated the fungal isolate had high sequence similarity with $O$. longissima (EF468865, 99\%), O. sobolifera (EF468875, 94\%), C. brunneipunctata (DQ522369, 90\%), C. agriota (DQ522368, 86\%) and O. rhizoidea (EF468873, 85\%). A phylogenetic tree was generated from 14 aligned sequences with an equal character. This tree indicated the fungal isolate was located in the same clade as $O$. longissima (Figure 4).

\section{Medium screening for fungal growth}

Seven media were screened to determine the optimum growth medium for the fungal isolate Cod-MK1. Colonies of the isolate grew to $69.5 \pm 0.5,67.5 \pm 0.5$ and $66.5 \pm 0.5$ in diameter on the HCGA, MEA and YMA media, respectively, over 20 days under 25 to $28^{\circ} \mathrm{C}$. Growth on HCGA was fast, with significant difference greater than that on the other media. On the other hand, growth on OMA and SDA media was particularly slow and significantly slower than on CDA and PDA media (Figure 5)

The mycelium obtained on the PDA, HCGA, OMA and SDA media agglutinated tightly and was of white cream color. The mycelium obtained on the YMA, MEA and CDA media was loose but had the same white cream color (Figure 6). 


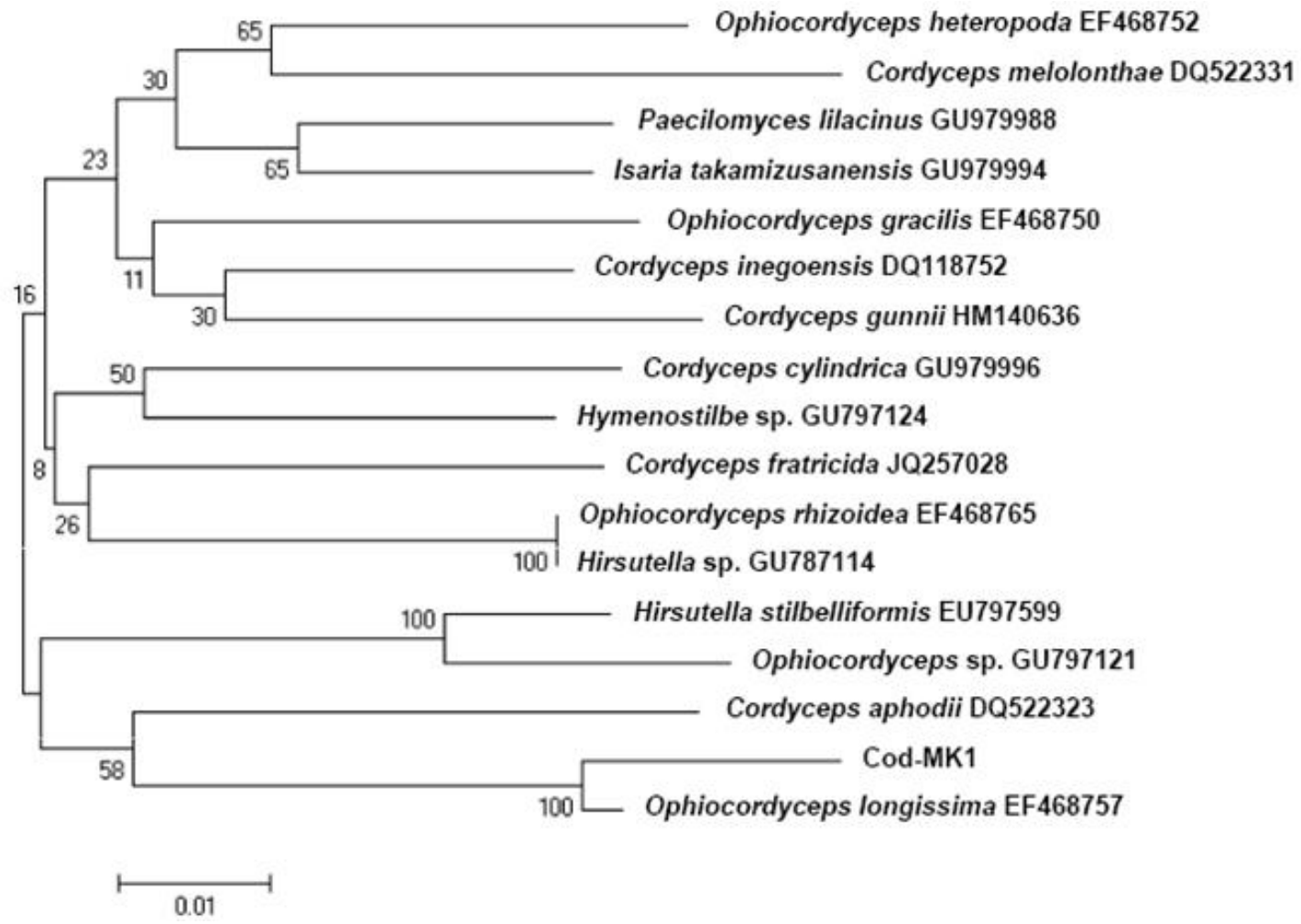

Figure 3. Phylogenetic relationships of the fungal isolate Cod-MK1 with Cordyceps and related species based on partial sequencing of the elongation factor $1 \alpha(E F-1 \alpha)$. A Neighbor Joining tree (NJ tree) was constructed using Mega 4. The percentages expressed above the branches are frequencies with which a given branch appeared in 1000 bootstrap replications.

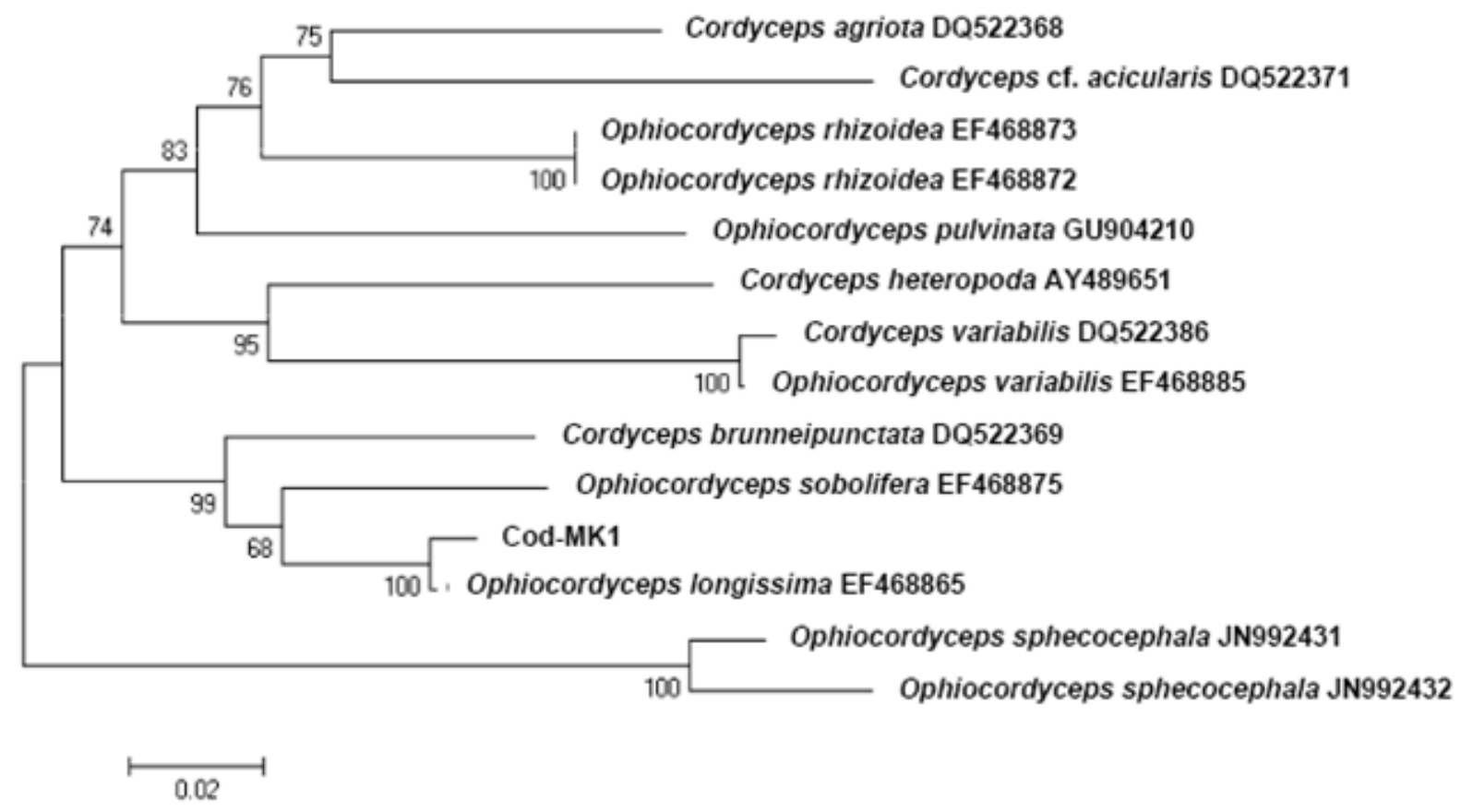

Figure 4. Phylogenetic relationships of the fungal isolate Cod-MK1 with Cordyceps and related species based on partial sequencing of the largest subunit of RNA polymerase II (rpb1). A Neighbor Joining tree (NJ tree) was constructed using Mega 4. The percentages expressed above the branches are frequencies with which a given branch appeared in 1000 bootstrap replications. 


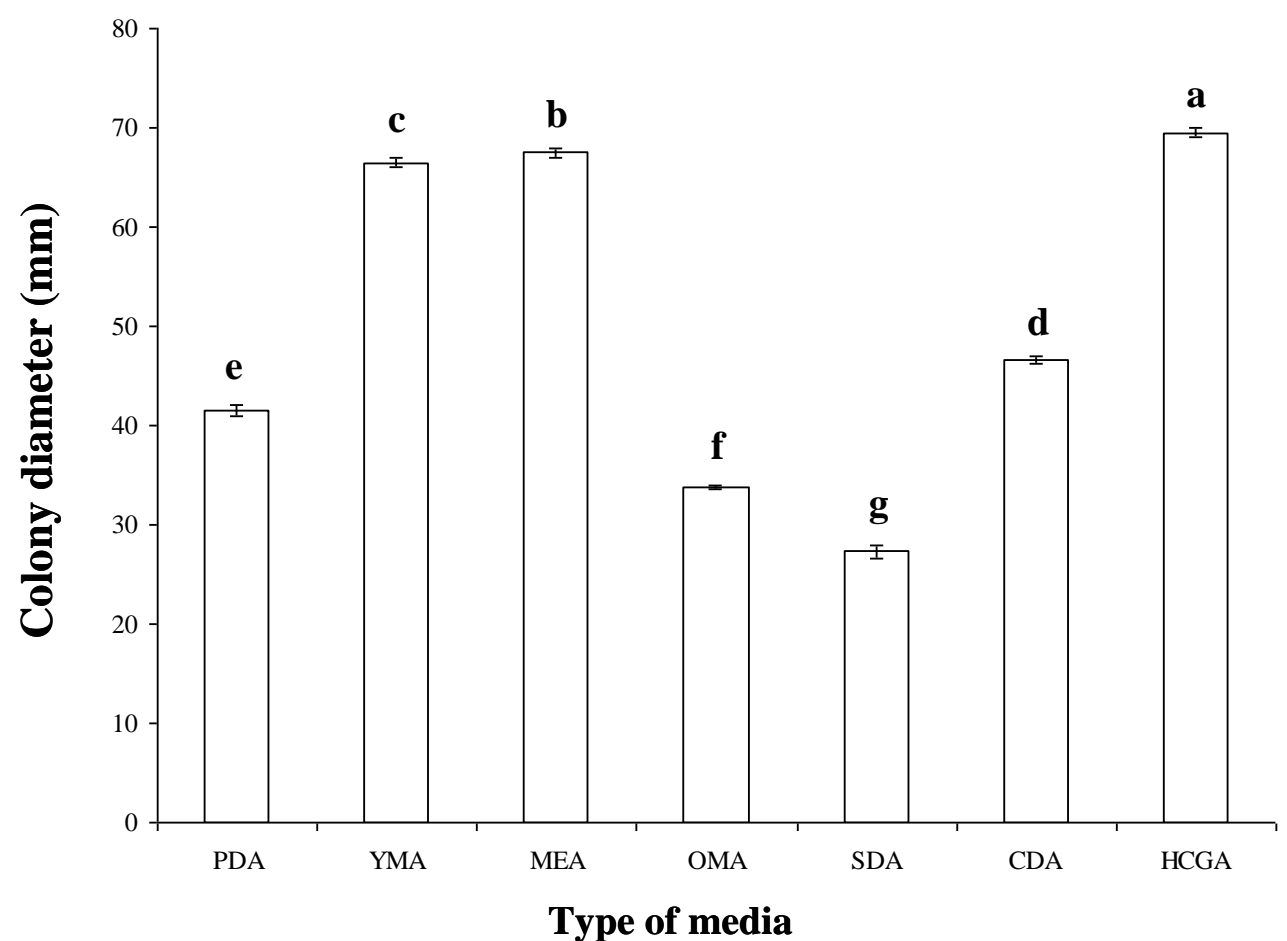

Figure 5. The mycelial growth of the fungal isolate Cod-MK1 on different media following 20 days incubation at ambient temperature. Values presented are the mean and standard error of three replicate plates. Values labeled with the same letter are not significantly different by Duncan's multiple range test at $P=0.05$.

\section{Induction of synnemata and conidia on applied HCGA medium}

The sterilized applied HCGA medium was inoculated with the fungal isolate Cod-MK1 and incubated at 25 to $28^{\circ} \mathrm{C}$. Synnemata formation first occurred on the surface of the medium after 45 to 60 days cultivation. Numerous synnemata were observed after 90 days. Conidia varied in size from 2 to $3 \times 8$ to $10 \mu \mathrm{m}$, and were produced by the mature synnemata or mycelium after over 100 days incubation (Figure 7).

\section{Determination of adenosine and cordycepin in the fungal isolate Cod-MK1}

HPLC analysis showed peaks of adenosine and cordycepin at the retention times of $9.57 \mathrm{~min}$ and 14.12 min, respectively. The extract from dried mycelia of the fungal isolate Cod-MK1 showed two peaks corresponding to adenosine and cordycepin (9.43 and $13.86 \mathrm{~min}$ ) and the extract from dried stroma-like stalks showed one peak of cordycepin at a retention time of $13.84 \mathrm{~min}$. Standard adenosine concentration ranging from 1 to 25 $\mu \mathrm{g} / \mathrm{mL}$ and cordycepin concentration ranging from 6.25 to $75 \mu \mathrm{g} / \mathrm{mL}$ were used to establish the linear curve between concentration and peak area. The regression equations were calculated as $Y=290.04 X-90.025\left(R^{2}=\right.$ $0.9992)$ for adenosine and $Y=46.739 X-85.697\left(R^{2}=\right.$ $0.9991)$ for cordycepin. These were established in triplicate. The content of adenosine was observed in the extract from dried mycelia at $31.68 \mu \mathrm{g} / \mathrm{g}$. The content of cordycepin from dried mycelia was $335.65 \mu \mathrm{g} / \mathrm{g}$; lower than the extract from dried stroma-like stalks (366.14 $\mu \mathrm{g} / \mathrm{g})$.

\section{DISCUSSION}

Appropriate culture medium nutrients are an important requirement for fungal growth. In this study, we successfully isolated the fungal isolate Cod-MK1 from the inner tissue of cicada larvae, but the growth rate was slow and culture took 30 days on the isolating medium. The slow growth rate on a culture medium lacking some special ingredients such as SDA, CDA and OMA was also found in this study. The growth rate of the fungal isolate Cod-MK1 on agar supplement with homogenized died cricket (HCGA medium) was faster than the other culture media. In addition, growth of the isolate on HCGA media was 1.7, 2.0, 2.5 and 1.5 times higher than on PDA, OMA, SDA and CDA media, respectively. These 

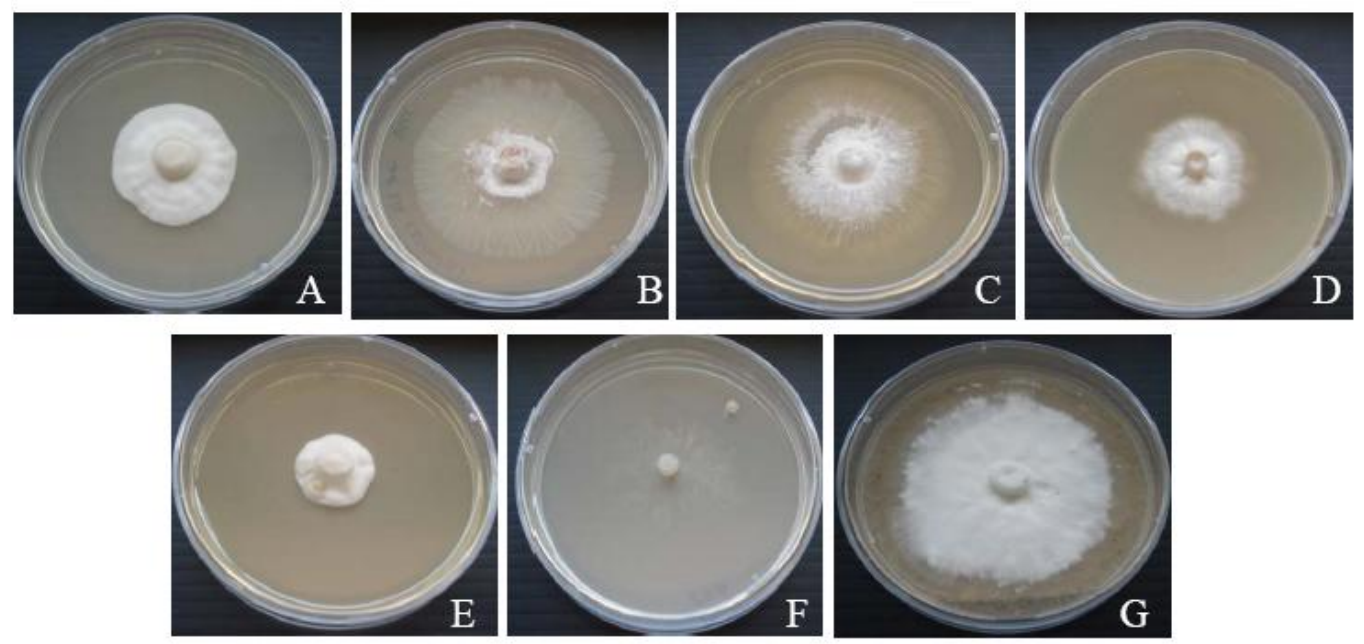

Figure 6. Various colony morphologies of the fungal isolate Cod-MK1 on six synthetic media (A-F) and one composed medium $(G)$ after 20 days incubation at ambient temperature. $A=P D A ; B=Y M A ; C=$ $M E A ; D=O M A ; E=S D A ; F=C D A ; G=$ HCGA.
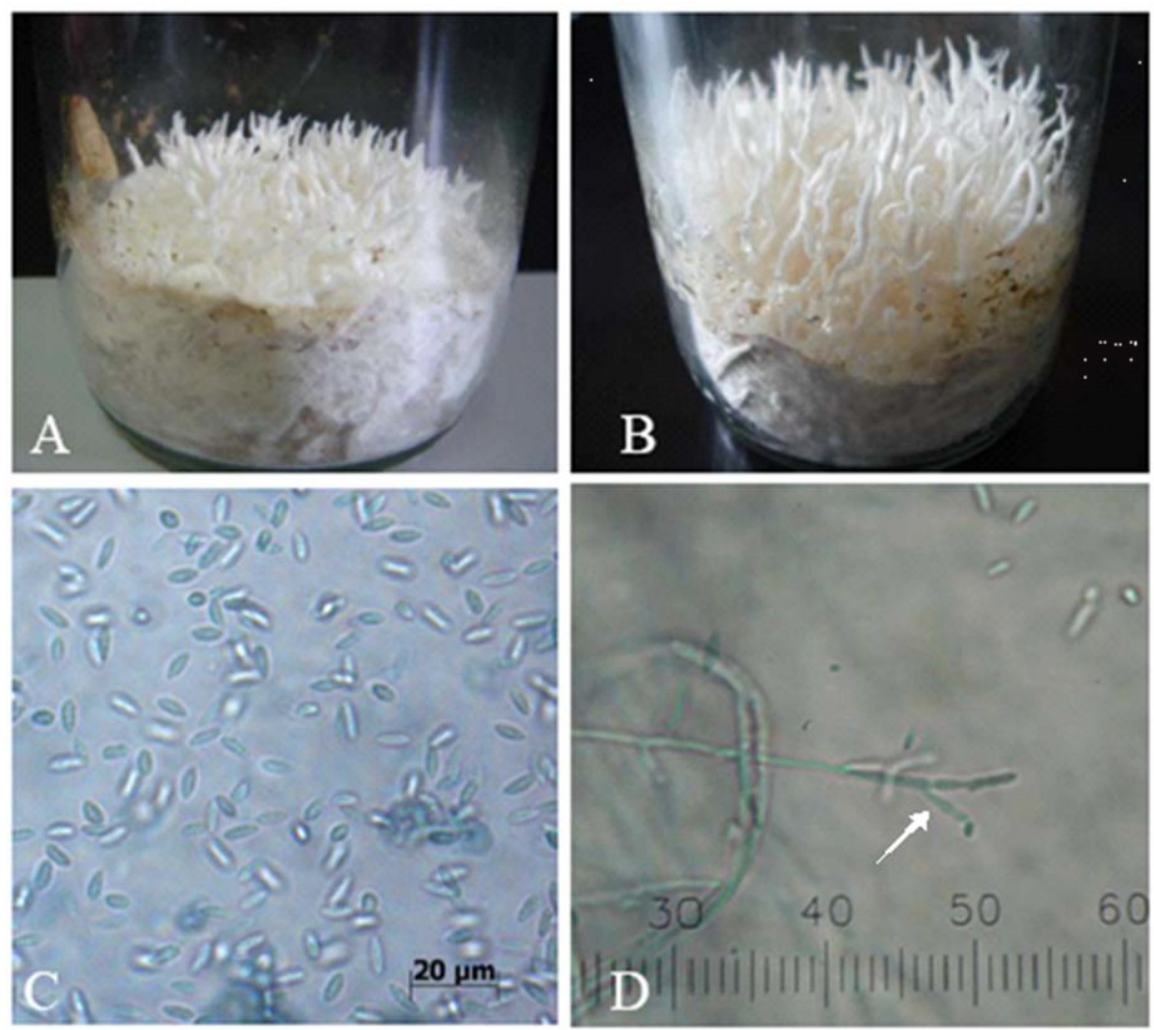

Figure 7. The fungal isolate Cod-MK1. White cream synnemata or cotton-like hyphae on applied HCGA medium. A, 60 days; B, 90 days after inoculation. C, conidia; D, phlialide (indicated by arrow).

results are in general agreement with those of Nam et al. (2006), who found that $C$. sphecocephala grew more slowly on PDA medium than PDBLA or PDBAA media containing of frozen dried honeybee larvae and honeybee adult. Supplementing media with mineral salts has also been shown to increase growth rate of $C$. cardinalis (Sung et al., 2010).

The fungal isolate Cod-MK1 was found to grow on 
applied HCGA. In this medium, the fungal isolate used Thai Jasmine rice as a carbon source, and homogenized died cricket as a nitrogen and mineral source. However, growth is slow. On this medium, numerous synnemata (stroma-like stalks) and conidia were only produced after 60 to 90 days incubation. Since Cordyceps species grow slowly, the isolating medium and culture medium should be supplemented with special ingredients to reduce isolation time, increase growth rate, and increase production of synnemata and conidia. Mycelium growth rate can also be increased by successive subculture (Sung et al., 2010).

In the present study, the phylogenetic relationship between the Cordyceps and related species based on SSU, LSU, EF-1 $\alpha$ and rpb1 gene were also investigated. The SSU rDNA phylogeny shows that the fungal isolate Cod-MK1 was not located within a clade with the other 13 isolates used, but shared a closer relationship with $O$. sobolifera and $C$. sobolifera. Similar findings were obtained from phylogeny derived from the LSU rDNA data set. In the LSU rDNA tree, the fungal isolate CodMK1 did not nest to the other 13 isolates, but classified in a clade with $H$. stilbelliformis var. Myrmicarum (anamorph of Ophicordyceps genus). Moreover, the EF-1 $\alpha$ and rpb1 phylogenetic tree showed that the fungal isolate CodMK1 sequences were closely related with $O$. longissima which $99 \%$ identity whereas other anamorphs and telomorphs of the genus Cordyceps showed a maximum identity of less than $95 \%$. Based on the nucleotide sequence of four genes, insect host and fungal morphology, the fungus isolated from the inner tissue of the cicada larvae can be identified as an anamorph strain of Ophiocordyceps and assigned as $\mathrm{O}$. longissima isolate Cod-MK1. These investigative tool resemble those of Chan et al. (2011), who used the sequence of the ITS region and three loci, nuclear ribosomal large subunit (nrLSU), elongation factor1 $\alpha(E F-1 \alpha)$ and the largest subunit of RNA polymerase II (rpb1) to identify Cordyceps samples as C. gunnii. Therefore, the SSU, LSU, EF-1 $\alpha$ and rpb1 primers represent beneficial tools for the classification and identification of Cordyceps species.

The ITS region, in particular, is very useful for species identification (Chen et al., 2001). This is because of the high diversity of the ITS region between species, as well as the homogeneity of the region within species. Indeed, the ITS region may be the most suitable marker to study the life cycles of Cordyceps sinensis, Hirsutella sinensis, Paecilomyces sp., Tolypocladium sp. and Stachybotrys sp. and confirm teleomorph-anamorph connection (Chen et al., 2001). Kuo et al. (2005) successfully used PCRsingle-strand conformation polymorphism (PCR-SSCP) of the ITS2 region for intraspecies classification of 17 Cordyceps isolates.

In this study, the contents of adenosine and cordycepin were measured using HPLC. The peaks corresponding to standard adenosine and cordycepin were identified.
These results indicate that $O$. longissima isolate CodMK1 can produce adenosine and cordycepin especially in induced conditions resembling those of $C$. militaris (Huang et al., 2009; Xie et al., 2009). However, the concentrations of adenosine and cordycepin produced in this study were low. Large scale production of adenosine and cordycepin using the O. longissima isolate Cod-MK1 would therefore require optimization of conditions.

In conclusion, this study isolated an entomopathogenic fungus and based on SSU, LSU, EF-1 $\alpha$ and rpb1 sequences analyses, identified it as belonging to the Ophiocordyceps genus. The isolate that grows best in HCGA medium, can develop numerous synnemata and conidia in applied HCGA medium, and can produce adenosine and cordycepin in induced condition.

\section{ACKNOWLEDGEMENTS}

We would like to thank Mahasarakham University Faculty of Science for the use of the equipment employed in this study. We would also like to thank Dr. Tim Cushnie for useful suggestions on the manuscript.

\section{REFERENCES}

Castlebury LA, Rossman AY, Sung GH, Hyten AS, Spatafora JW (2004). Multigene phylogeny reveals new lineage for Stachybotrys chartarum, the indoor air fungus. Mycol. Res. 108:864-872.

Chan WH, Ling KH, Chiu SW, Shaw PC, But PPH (2011). Molecular analyses of Cordyceps gunnii in China. J. Food Drug Anal. 19(1):1825.

Chen YQ, Wang N, Qu LH, Li TH, Zhang WM (2001). Determination of anamorph of Cordyceps sinensis inferred from the analysis of the ribosomal DNA internal transcribed spacers and 5.8S rDNA. Biochem. Sys. Ecol. 29:597-607.

Currie CR, Wong B, Stuart AE, Schultz TR, Rehner SA, Mueller UG, Sung GH, Spatafora JW, Straus NA (2003). Ancient tripartite coevolution in the attine ant-microbe symbiosis. Science 299:386-388.

Das SK, Masuda M, Sakurai A, Sakakibara M (2010). Medicinal uses of the mushroom Cordyceps militaris: current state and prospects. Fitoterapia 81:961-968.

Dong $\mathrm{CH}$, Yao YJ (2005). Nutritional requirements of mycelial growth of Cordyceps sinensis in submerged culture. J. Appl. Microbiol. 99:483492.

Holliday J, Cleaver M (2008). Medicinal value of the caterpillar fungi species of the genus Cordyceps (Fr.) Link (ascomycetes): a review. Int. J. Med. Mushrooms 10:219-234.

Huang L, Li Q, Chen Y, Wang X, Zhou X (2009). Determination and analysis of cordycepin and adenosine in the products of Cordyceps spp. Afr. J. Microbiol. Res. 3(12):957-961.

Hung LT, Keawsompong S, Hanh VT, Sivichai S, Hywel-Jones NL (2009). Effect of temperature on cordycepin production in Cordyceps militaris. Thai. J. Agric. Sci. 42(4):219-225.

Kiho T, Kaizu Y, Taketomo N, Tusunoo A (1996). Physiological activities of the extracts from cultured mycelia of Cordyceps militaris (Vuill.) Fr Bull. Gen. Educ. Tokyo Med Dent Univ. 26:7-14.

Kuo HC, Su YL, Yang HL, Chen TY (2005). Identification of Chinese medicinal fungus Cordyceps sinensis by PCR-single-stranded conformation polymorphism and phylogenetic relationship. J. Agric. Food Chem. 53:3963-3968.

Leung PH, Zhao S, Ho KP, Wu JY (2009). Chemical properties and antioxidant activity of exopolysaccharides from mycelial culture of Cordyceps sinensis fungus Cs-HK1. Food Chem. 114:1251-1256.

Liu ZY, Yao YJ, Liang ZQ, Liu AY, Pegler DN, Chase MW (2001). 
Molecular evidence for the anamorph-teleomorph connection in Cordyceps sinensis. Mycol. Res. 105:827-832.

Masuda M, Urabe E, Sakurai A, Sakakibara M (2006). Production of cordycepin by surface culture using the medicinal mushroom Cordyceps militaris. Enzyme Microb. Technol. 39:641-646.

Matsuda S, Takamatsu S (2003). Evolution of host-parasite relationships of Golovinomyces (Ascomycete: Erysiphaceae) inferred from nuclear rDNA sequence. Mol. Phylogenet. Evol. 27:314-327.

Nam SH, Li CR, Hong IP, Sung KB, Kang SW, Fan MZ, Li ZZ (2006). Isolation and culture of entomopathogenic fungus, Cordyceps sphecocephala. Int. J. Ind. Entomol. 13(2):57-61.

Ohmori T, Tamura K, Tsuru S, Nomoto K (1986). Antitumor activity of protein-bound polysaccharide from Cordyceps ophioglossoides in mice. Jpn. J. Cancer Res. (Gann), 77:1256-1263.

Paterson RR (2008). Cordyceps: a traditional Chinese medicine and another fungal therapeutic biofactory? Phytochemistry 69:1469-1495.

Skouboe P, Frisvad JC, Taylor JW, Lauritsen D, Boysen M, Rossen L (1999). Phylogenetic analysis of nucleotide sequences from the ITS region of terverticillate Penicillium species. Mycol. Res. 103:873-881.

Sung GH, Shrestha B, Han SK, Kim SY, Sung JM (2010). Growth and cultural characteristics of Cordyceps cardinalis collected from Korea. Mycobiology 38(4):274-281.

Tamura K, Dudley J, Nei M, Kumar S (2007). MEGA4: molecular evolutionary genetics analysis (MEGA) software version 4.0. Mol. Biol. Evol. 24:1596-1599.

Vilgalys R, Hester M (1990). Rapid genetic identification and mapping of enzymatically amplified ribosomal DNA from several Cryptococcus species. J. Bacteriol. 172:4238-4246.

Webster J (1980). Introduction to Fungi. $2^{\text {nd }}$ Edn., Cambridge University Press, Cambridge, p. 355.
Wang Y, Guo Y, Zhang L, Wu J (2012). Characterizations of a new Cordyceps cicadae isolate and production of adenosine and cordycepin. Braz. J. Microbiol. pp. 449-455.

Weng SC, Chou CJ, Lin LC, Tsai WJ, Kuo YC (2002). Immunomodulatory functions of extracts from the Chinese medicinal fungus Cordyceps cicadae. J. Ethnopharmacol. 83:79-85.

White TJ, Bruns T, Lee S, Taylor J (1990). Amplification and direct sequencing of fungal ribosomal RNA genes for phylogenetics. In PCR protocols, a guide to methods and applications; Innis MA, Gelfand DH, Sninsky JJ, White TJ (Eds). Academic Press: San Diego: pp. 315-322.

Wu F, Yan H, Ma X, Jia J, Zhang G, Guo X, Gui Z (2011). Structural characterization and antioxidant activity of purified polysaccharide from cultured Cordyceps militaris. Afr. J. Microbiol. Res. 5(18):27432751.

Xie CY, Gu ZX, Fan GJ, Gu FR, Han YB, Chen ZG (2009). Production of cordycepin and mycelia by submerged fermentation of Cordyceps militaris in mixture natural culture. Appl. Biochem. Biotechnol. 158:483-492.

Zhu JS, Halpern GM, Jones K (1998). The scientific rediscovery of an ancient Chinese herbal medicine: Cordyceps sinensis. Part I. J. Altern. Complement Med. 4:289-303.

Zhu R, Chen YP, Deng YY, Zheng R, Zhong YF, Wang L, Du LP (2011). Cordyceps cicadae extracts ameliorate renal malfunction in a remnant kidney model. Zhejiang Univ-Sci B (Biomed \& Biotechnol), 12(12):1024-1033. 